\title{
Internal Carotid Artery Dissection Causing Lower Cranial Neuropathies
}

\author{
Clare Perkins $^{1 *}$, Mihiar Atfeh ${ }^{2}$ and Mark Medcalf ${ }^{3}$ \\ ${ }^{1}$ Core Trainee in ENT Surgery, Derriford Hospital, UK \\ ${ }^{2}$ Clinical Fellow in ENT Surgery, Derriford Hospital, UK \\ ${ }^{3}$ Consultant in ENT Surgery, Derriford Hospital, UK
}

*Corresponding author: Clare Perkins, ENT Department, Derriford Hospital, UK.

Received Date: January 22, 2019

Published Date: February 11, 2019

\begin{abstract}
As a recognised cause of acute stroke, particularly in younger patients, spontaneous carotid artery dissection is an important diagnosis to consider. Although advances in interventional radiology are improving diagnosis, it is still considered an underrecognised phenomenon [1]. It is estimated to account for around $2.5 \%$ of all strokes, but this figure is much higher for younger patients [2]. However, the clinical presentation can be varied, subtle and often challenging to make, with multiple different presentations described. Unilateral cranial nerve palsies, particularly of the lower cranial nerves, are a recognised sign in up to $12 \%$ of cervical artery dissections [1], and it is therefore vital to be able to recognise them. We present a case of a man referred to a head and neck cancer clinic with unilateral lower cranial neuropathies and neck pain who was subsequently found to have an internal carotid artery dissection.
\end{abstract}

Keywords: Cranial nerves; Carotid artery dissection; Stroke

Abbreviations: MRI: Magnetic Resonance Imaging; CTA: Computed Tomography Angiography

\section{Introduction}

As a recognised cause of acute stroke, particularly in younger patients, spontaneous carotid artery dissection is an important diagnosis to recognise. Although advances in interventional radiology are improving diagnosis, it is still considered an underrecognised phenomenon [1]. It is estimated to account for around $2.5 \%$ of all strokes, but this figure is much higher for younger patients [2]. However, clinical presentation can be varied, subtle and often challenging to make, with many different presentations described. The most commonly described symptom is pain in either the head, neck or face, and tends to be unilateral [3]. Partial Horner's syndrome is also a common symptom [4], found in up to $50 \%$ of patients [3], as well as symptoms secondary to cerebral ischaemia. However, there are several less common symptoms associated with spontaneous carotid artery dissection which many different specialties should be able to recognise, including cranial nerve palsies. Unilateral cranial nerve palsies, particularly of the lower cranial nerves, are a recognised sign in up to $12 \%$ of cervical artery dissections [1].

\section{Case}

A man in his 70s presented to the Head and Neck two-weekwait clinic with a 3-week history of right sided neck pain, right sided unilateral sore throat and otalgia, nasal speech and dysphagia. The symptoms began rapidly and had begun to improve by the time of the appointment. He had had no trauma and remained well in himself with no fevers or weight loss.

His past medical history included ischemic heart disease and peripheral vascular disease. He was a non-smoker.

Examination of the oral cavity and oropharynx revealed tongue fasciculations and deviation of the tongue and uvula to the right. He was unable to move his tongue to the left and had reduced palatal movement on the right. There was no cervical lymphadenopathy. Flexible nasendoscopy was normal, revealing no masses or vocal cord palsy.

He underwent a Magnetic Resonance Imaging (MRI) scan with contrast (Figure 1) which revealed contrast within the sub-intimal 
layer of the right internal carotid artery in keeping with an internal carotid artery dissection. He was subsequently referred to the vascular team and underwent Computer Tomography Angiography (CTA) to further assess the dissection.

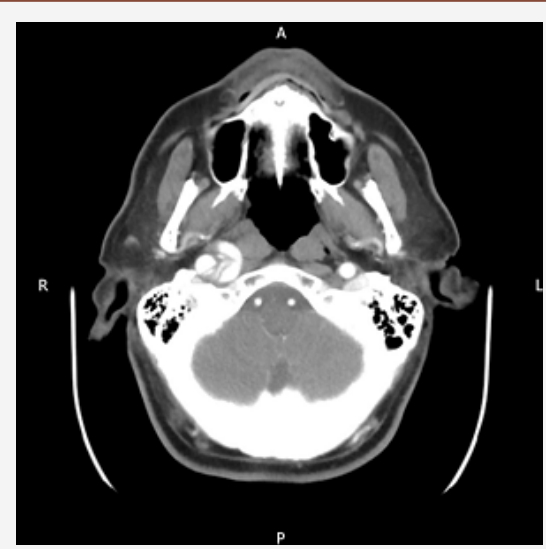

Figure 1: Axial computed-tomography angiography image at the level of the skull base depicting a right internal carotid artery dissection with contrast seen within the vessel wall.

He was managed conservatively with anti-platelet drugs and interval scanning, and at one-year post follow-up he is symptom free, although the dissection is still present on repeat CTA.

\section{Discussion}

Spontaneous cervical arterial dissection occurs when circulating blood enters the vessel wall without preceding trauma causing a mural haematoma [5]. This can lead to a dissecting aneurysm thus increasing the diameter of the vessel wall which can cause compression of surrounding structures. The underlying pathophysiology is not entirely understood, but factors just as infection, minor trauma and environmental factors such as underlying vessel wall weakness are considered potential contributing factors $[5,6]$.

Cranial nerve palsies are reported in up to $12 \%$ of cases of spontaneous carotid artery dissection, with the most common nerve affected being the hypoglossal [1].

Where cranial nerve lesions occur, the lower cranial nerves are more commonly affected. Possible causes include direct compression from the dissecting aneurysm vessel close to its entrance at the skull base and interrupted blood flow to the nerves. The internal carotid artery enters the skull base through the carotid canal along with sympathetic nerve fibres. The inferior entrance to carotid canal lies close to the jugular foramen, through which the glossopharyngeal, vagus and spinal accessory nerves (Cranial Nerves IX - XI) exit the skull. It also lies medially to the hypoglossal canal, transmitting the hypoglossal nerve.

Recognition of clinical signs associated with lower cranial nerve lesions is vital in the detection of a potential cervical artery dissection and may help to avert an acute stroke. Signs such as reduced palate sensation (glossopharyngeal nerve), reduced gag reflex (vagus and glossopharyngeal nerves) breathy dysphonia due to unilateral vocal cord palsy (vagus nerve), inability to shoulder raise on the affected side (spinal accessory nerve) and unilateral tongue paralysis with deviation of the tongue towards the affected side and tongue fasciculations (hypoglossal nerve) point to lower cranial nerve lesions [3]. Lower cranial neuropathies can be symptom of many conditions, often affecting the skull base [3], but in conjunction with unilateral neck, head or facial pain could indicate a cervical artery dissection.

\section{Acknowledgement}

None

\section{Conflict of interest}

No conflict of interest

\section{References}

1. Mokri B, Sundt T, Wayne O, Piepgras D (1986) Spontaneous dissection of the cervical internal carotid artery. Ann Neurol 19(2): 126-138.

2. Blunt S, Galton C (1997) Cervical carotid or vertebral artery dissection: An underdiagnosed cause of stroke in the young. BMJ 314: 243.

3. Finsterer J, Grisold W (2015) Disorders of the lower cranial nerves. J Neurosci Rural Pract 6(3): 377-391.

4. Guillon B, Lévy C, Bousser MG (1998) Internal carotid artery dissection: an update. J Neurol Sci 153(2):146-158.

5. Guillon B, Bousser MG (2002) Epidemiology and pathophysiology of spontaneous cervical artery dissection. J Neuroradiol. 29(4): 241-249.

6. Debette S (2014) Pathophysiology and risk factors of cervical artery dissection: what have we learnt from large hospital-based cohorts? Curr Opin Neurol 27(1): 20-28. 